\title{
Construction of a long non-coding RNA-mediated transcription factor and gene regulatory triplet network reveals global patterns and biomarkers for ischemic stroke
}

\author{
YUZE CAO $^{1,2}$, JIANJIAN WANG ${ }^{2}$, XIAOYU LU ${ }^{2}$, XIAOTONG KONG ${ }^{2}$, CHUNRUI BO $^{2}$, SHUANG LI ${ }^{2}$,

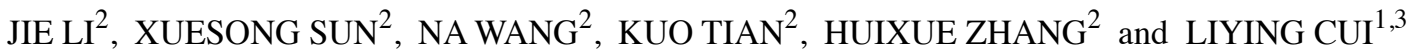 \\ ${ }^{1}$ Department of Neurology, Peking Union Medical College Hospital, Chinese Academy of Medical Sciences, \\ Beijing 100730; ${ }^{2}$ Department of Neurology, The Second Affiliated Hospital, Harbin Medical University, Harbin, \\ Heilongjiang 150086; ${ }^{3}$ Neuroscience Center, Chinese Academy of Medical Sciences, Beijing 100730, P.R. China
}

Received January 5, 2019; Accepted August 20, 2019

DOI: $10.3892 /$ ijmm.2019.4421

\begin{abstract}
Ischemic stroke (IS) is a severe neurological disease and a major cause of death and disability throughout the world. A long non-coding (lnc)RNA, transcription factor (TF) and gene can form a lncRNA-mediated regulatory triplet (LncMRT), which is a functional network motif that regulates numerous aspects of human diseases. However, systematic identification and molecular characterization of LncMRTs and their roles in IS has not been carried out. In the present study, a global LncMRT network was constructed and the topological features were characterized based on experimentally verified interactions. An integrated approach was developed to identify significantly dysregulated LncMRTs in peripheral blood mononuclear cells of IS and these dysregulated LncMRT networks exhibited specific topological characteristics and a closer network structure than the global LncMRT network that was constructed. The variation of the risk score for LncMRTs indicated that there were multiple dysregulated patterns of LncMRTs in IS. Numerous core clusters were identified from dysregulated LncMRT networks and these core clusters could distinguish IS patient and matched control samples. Finally, functional analyses demonstrated that LncMRTs associated with IS participated in the regulation of the phosphatidylinositol 3-kinase/protein kinase B signaling pathway. In conclusion, the roles of the LncMRTs in IS were
\end{abstract}

Correspondence to: Professor Liying Cui, Department of Neurology, Peking Union Medical College Hospital, Chinese Academy of Medical Sciences, Beijing 100730, P.R. China

E-mail: pumchcuily@sina.com

Dr Huixue Zhang, Department of Neurology, The Second Affiliated Hospital, Harbin Medical University, 246 Xuefu Road, Harbin, Heilongjiang 150086, P.R. China

E-mail: zhanghuixue2009@163.com

Key words: long non-coding RNAs, ischemic stroke, biomarker, transcription factor, network elucidated, which could be beneficial for understanding IS pathogenesis and treatment.

\section{Introduction}

Ischemic stroke (IS) is a severe neurological disease and a major cause of death and disability throughout the world (1). Although rates of IS mortality and financial burden vary greatly among countries, low-income and middle-income countries are the most affected (2). Thrombotic stroke, embolic stroke, systemic hypoperfusion and venous thrombosis may lead to IS. IS has great heterogeneity since various pathophysiological mechanisms are usually involved (3). The application of unbiased genome-wide approaches has contributed to the understanding of IS. Quarta et al (4) investigated the relationship between cholesteryl ester transfer protein gene variants and the risk of IS. O'Connell et al (5) identified a pattern of gene expression in the peripheral blood of IS patients. However, the etiology of and mechanism underlying IS are unknown in most patients and identifying novel signatures or biomarkers that enhance clinical decisions in the treatment of IS are essential.

In addition to the construction of globally altered mRNA expression profiles in IS, the expression profiles of long non-coding (lnc)RNAs have also contributed to IS research (6). lncRNAs are defined as transcripts of more than 200 nucleotides that do not code for proteins and are pervasive across the genome (7). A previous study have demonstrated that the lncRNA expression profiles are altered in patient blood after IS and several lncRNAs have been shown to play roles in animal models of stroke and in vitro models of oxygen-glucose deprivation (8). Zhu et al (9) suggested that the lncRNA H19 rs217727 gene polymorphism contributes to IS susceptibility and may serve as a potential indicator for IS susceptibility. lncRNA rhabdomyosarcoma 2 associated transcript silencing has been shown to protect against middle cerebral artery occlusion-induced IS (10). However, studies into lncRNAs in IS are just beginning and little is known about their roles in IS. Numerous IncRNAs remain undiscovered in human IS and the functions of the majority of lncRNAs have not yet been elucidated. 
Transcription factors (TFs) determine the level of gene expression by recognizing specific DNA sequences in diverse cell types (11). TFs could also regulate the expression of lncRNAs in a number of diseases $(12,13)$. Regulatory relationships also exist between genes and lncRNAs $(14,15)$. LncRNAs, TFs and genes can form lncRNA-mediated regulatory triplets (LncMRTs), which have been widely observed in human diseases (16). However, the variety of LncMRT roles in IS has not been studied in a systematic manner.

In the present study, a global LncMRT network was constructed using experimentally verified TF-lncRNA, TF-gene and gene-lncRNA interactions. A dysregulatedLncMRT network for IS was also constructed using a comprehensive computational approach based on IncRNA, TF and gene expression profiles of IS patients. In LncMRT networks, IncRNAs showed specific topological characteristics similar to coding genes in IS. The dysregulated LncMRT network exhibited a closer network structure than the global LncMRT network. Several patterns in these dysregulated LncMRTs were found, including the absence and presence of regulatory relationships. Moreover, several core clusters were identified and these core clusters could distinguish between matched control and IS patient samples. Functional analyses revealed that dysregulated LncMRTs in IS participate in the regulation of gene expression and cell proliferation. The phosphatidylinositol 3-kinase (PI3K)/protein kinase B (Akt) signaling pathway was identified as an IS-associated pathway. In conclusion, the present study highlighted the effect of dysregulated LncMRTs in IS, which revealed their possibility as novel biomarkers and treatment targets in IS.

\section{Materials and methods}

Construction of an experimentally validated global LncMRT network. TF-lncRNA interaction data were download from SNP@lincTFBS, which identified the TF binding sites of lncRNAs using genome-wide chromatin immunoprecipitation sequencing data (17). TF-gene interaction data were obtained from TRANScription FACtor database (18). Gene-lncRNA interaction data were obtained from RNA Association Interaction Database, which integrates experimental gene-lncRNA interactions from manually reading the literature and other database resources (19). Then the global LncMRT network was constructed following the aforementioned experimentally validated interactions.

Expression profiles of TFs, genes and lncRNAs for IS. LncRNA, TF and gene expression profiles of IS patients and matched controls were downloaded from the Gene Expression Omnibus database (www.ncbi.nlm.nih.gov/geo). The study selected contained peripheral blood mononuclear cells of 20 IS patients and 20 sex- and age-matched controls (GSE22255) (20). The IS patients in the aforementioned study suffered only one stroke episode $\geq 6$ months before the blood collection and controls could not have a family history of stroke. The authors also excluded participants with severe anemia or active allergies (Table SI).

Identification of dysregulated LncMRTs in IS. A comprehensive computational approach was developed to identify significantly dysregulated LncMRTs in IS based on the global
LncMRT network and expression data. Initially, a t-test was used to identify the differential level of expression of the lncRNA, TF and gene in each LncMRT; the t-test compared the expression levels between IS patients and matched controls, resulting in P-values. Second, Pearson Correlation Coefficients (PCCs) were calculated for each interacting pair (TF-lncRNA, TF-gene and lncRNA-gene) in a LncMRT based on the expression profile of IS patients. Different PCCs were represented using the absolute difference of PCCs between the IS patients and matched controls. Third, an integrated approach was performed based on two comprehensive risk scores, including differential expression P-values $\left(\mathrm{RS}_{\mathrm{dif}}\right)$ and PCCs $\left(\mathrm{RS}_{\mathrm{PCC}}\right)$ for each LncMRT as follows:

$$
\begin{gathered}
R S_{\mathrm{dif}}=P_{\mathrm{T}} x P_{\mathrm{L}} \times P_{\mathrm{G}} \\
R S_{\mathrm{PCC}}=\left[\left(I S_{\mathrm{TL}}-C O N_{\mathrm{TL}}\right) x\left(I S_{\mathrm{TG}^{-}}-C O N_{\mathrm{TG}}\right) x\left(I S_{\mathrm{GL}^{-}}-C O N_{\mathrm{GL}}\right)\right]
\end{gathered}
$$

where $\mathrm{P}_{T}, \mathrm{P}_{\mathrm{L}}, \mathrm{P}_{\mathrm{G}}$ represented the P-values of the differentially expressed TF, IncRNA and gene, respectively, in each LncMRT. The integral differential expression level between IS patient and matched control samples of a LncMRT was indicated by $\mathrm{RS}_{\mathrm{dif}}$. IS $\mathrm{IL}_{\mathrm{TL}}, \mathrm{IS}_{\mathrm{TG}}$ and $\mathrm{IS}_{\mathrm{GL}}$ represented the PCCs of interactions between the TF and lncRNA, the TF and gene, and the lncRNA and gene, respectively in IS patients. $\mathrm{CON}_{\mathrm{TL}}$, $\mathrm{CON}_{\mathrm{TG}}$ and $\mathrm{CON}_{\mathrm{GL}}$ represented the PCCs of interactions between the TF and IncRNA, the TF and gene, and the IncRNA and gene, respectively in matched controls. The difference in the co-expression level of a LncMRT between IS patients and matched controls was indicated by $\mathrm{RS}_{\mathrm{PCC}}$.

Fourth, an equal-weighted multiple ranking approach was performed to rank all LncMRTs based on their $\mathrm{RS}_{\text {dif }}$ and $\mathrm{RS}_{\mathrm{PCC}}$. After this step, each LncMRT received a final risk score and was ranked by these final risk scores. Finally, 1,000 sample labels of the expression profiles were randomly permuted to compare the final risk score with the permutation risk score and obtain significant results. A permutation result of $\mathrm{P}<0.05$ was selected as the threshold value to generate significantly dysregulated LncMRTs for IS.

Topological features of the global LncMRT network and the dysregulated LncMRT network for IS. Topological features including degree, clustering coefficient and network density analyses were performed for all the nodes in the two networks using Cytoscape 3.0 (http://www.cytoscape.org/).

Identification of core clusters from the dysregulated LncMRT network for IS. Core clusters were extracted from the dysregulated LncMRT network for IS using the Clustering with Overlapping Neighborhood Expansion (ClusterONE) package in Cytoscape, with default parameters (http://apps.cytoscape. org/apps/ClusterONE). ClusterONE is a package that clusters a given network based on topology to identify densely connected regions. Finally, four core clusters were extracted based on the number of nodes and the cluster scores.

Classification power of the core clusters in IS. A consensus clustering approach was used to classify 20 IS patients and 20 matched controls based on expression data of TFs, genes and IncRNAs (21). The ConsensusClusterPlus package in $\mathrm{R}$ (https://www.r-project.org/) was used to perform this process. 
The smallest increase in the area under the cumulative distribution function (CDF) curve was defined as the best category number. A Chi-square test was applied to evaluate whether IS patients and matched controls could be classified using this method $(\mathrm{P}<0.05)$.

Functional enrichment analysis for dysregulated LncMRTs in IS. TFs and genes were selected for functional enrichment analyses to assess the functionality of the LncMRTs. Online Enrichr tool was applied with default parameters to perform the functional enrichment analyses (22). Enriched Gene Ontology (GO) terms and Kyoto Encyclopedia of Genes and Genomes (KEGG) pathways were identified using $\mathrm{P}<0.01$ and $\mathrm{P}<0.05$, respectively, as the thresholds.

\section{Results}

The construction of the global LncMRT network and topological analysis. A global LncMRT network was constructed using experimentally verified interactions (Fig. 1A). Details of the interactions are listed in Table SII. The global LncMRT network contained 4,554 LncMRTs, 1,153 nodes (including 74 TFs and genes, 501 genes, 524 lncRNAs, and 54 TFs) and 5,383 edges (Fig. 1B). The lncRNAs occupied a large proportion in all nodes and may play essential roles in the LncMRT network. The global LncMRT network exhibited scale-free distribution $\left(\mathrm{R}^{2}=0.728\right)$, which is a specific topological feature of transcriptional regulatory networks (Fig. 1C). Next, the authors found that the degrees of TFs, genes and lncRNAs approximated the scale-free network (Fig. 1D-F). Transcription factor E2F (E2F)1, E2F6 and E2F4 were the TFs with the highest degrees and are all members of the E2F family. The E2F family plays crucial roles in the control of the cell cycle and was also a target of transforming proteins of small DNA tumor viruses (23). Metastasis associated lung adenocarcinoma transcript 1 , taurine upregulated gene 1 and nuclear enriched abundant transcript 1 (NEAT1) were the lncRNAs with the highest degrees and were previously associated with IS $(24,25)$. Proto-oncogene c-Fos, myc proto-oncogene protein (MYC) and transcription factor Sp1 (SP1) were the genes with the highest degrees. These results indicated that the global LncMRT network could be useful background information to help design IS studies.

Specific LncMRTs are dysregulated in IS patients. An integrated computational pipeline was used to dissect the functional significance of LncMRTs in IS and 196 significantly dysregulated LncMRTs that were identified in IS $(\mathrm{P}<0.05)$. A significantly dysregulated LncMRT network was constructed and the network contained 183 nodes and 396 edges (Fig. 2A). There were eight TFs or genes, 21 TFs, 85 genes and 71 lncRNAs in the significantly dysregulated LncMRT network (Fig. 2B; Table SIII). The result indicated that lncRNAs occupied a large proportion in all nodes and may play essential roles in the LncMRT network; this phenomenon was similar to the global LncMRT network. Some of the lncRNAs in the dysregulated LncMRT network had been identified by other in vitro or mouse model studies (Table SIV). The degree of all nodes still approximated a scale-free network $\left(\mathrm{R}^{2}=0.603\right.$; Fig. 2C). The average degree of the significantly dysregulated
LncMRT network was smaller than the global LncMRT network and indicated the specific features of the LncMRTs (Fig. 2D). The dysregulated LncMRT network showed a higher clustering coefficient and network density compared with the global LncMRT network (Fig. 2E and F). The result indicated that the dysregulated LncMRT network had closer network structure features than the global LncMRT network and may be a functional network for IS.

Risk score profiles and multiple dysregulated patterns of LncMRTs in IS. Risk score profiles were created to characterize the compactness of each dysregulated LncMRT in IS (Fig. 3A). The two key risk score $\left(\mathrm{RS}_{\mathrm{dif}}\right.$ and $\mathrm{RS}_{\mathrm{PCC}}$ ) profiles were constructed and they showed similar unimodal distribution. The results indicated that the trends of the two risk scores were consistent. The global risk score profile was also constructed and it was discovered that the risk scores of most dysregulated LncMRTs were concentrated in a small scale. The detail risk score profile of top 10 dysregulated LncMRTs were identified and it was found that some risk scores did not change while others exhibited large changes (Fig. 3B). For instance, the difference in PCCs between IS patients and matched controls was bigger than the P-values in the dysregulated LncMRT SP1/long intergenic non-protein coding RNA (LINC)00475/interferon regulatory factor 4. However, the change in those P-values was larger compared with the PCC values in the dysregulated LncMRT SP1/UBAC2 antisense RNA 1 (UBAC2-AS1)/synaptic functional regulator FMR1 (FMR1). Therefore, it was inferred that there are several complex patterns of these dysregulated LncMRTs.

In the dysregulated LncMRT SP1/UBAC2-AS1/FMR1, the interaction between FMR1 and UBAC2-AS1 was absent in IS patients, and all the molecules were downregulated (Fig. 3C). In the dysregulated LncMRT trans-acting T-cell-specific transcription factor GATA-3 (GATA3)/colorectal neoplasia differentially expressed (CRNDE)/aurora kinase A (AURKA), the interaction between GATA3 and AURKA was present in IS patients (Fig. 3D). However, the other two interactions were both absent in IS patients. The TF GATA3 was upregulated and the gene AURKA and the lncRNA CRNDE were both downregulated. Collectively, the results show that interactions were absent or present in IS patients and demonstrate complex features.

IS-related core clusters could be regarded as specific biomarkers to distinguish IS patient and matched control samples. A core cluster analysis was performed to further reveal the communication between these three types of molecules in the dysregulated LncMRT network for IS. A total of four core clusters were identified and each core cluster contained a certain number of TFs, genes and lncRNAs (Fig. 4A). The authors also discovered that the last three core clusters shared most of their molecules and may have similar functions. The last three core clusters were combined to construct an integrated core cluster containing two TFs, one gene and five lncRNAs (Fig. 4B). The expression profiles of the TFs, genes and lncRNAs in each cluster were used to classify IS patient and matched control samples following consensus clustering, which was used to determine whether the LncMRTs could be regarded as special classifiers for IS. 
A
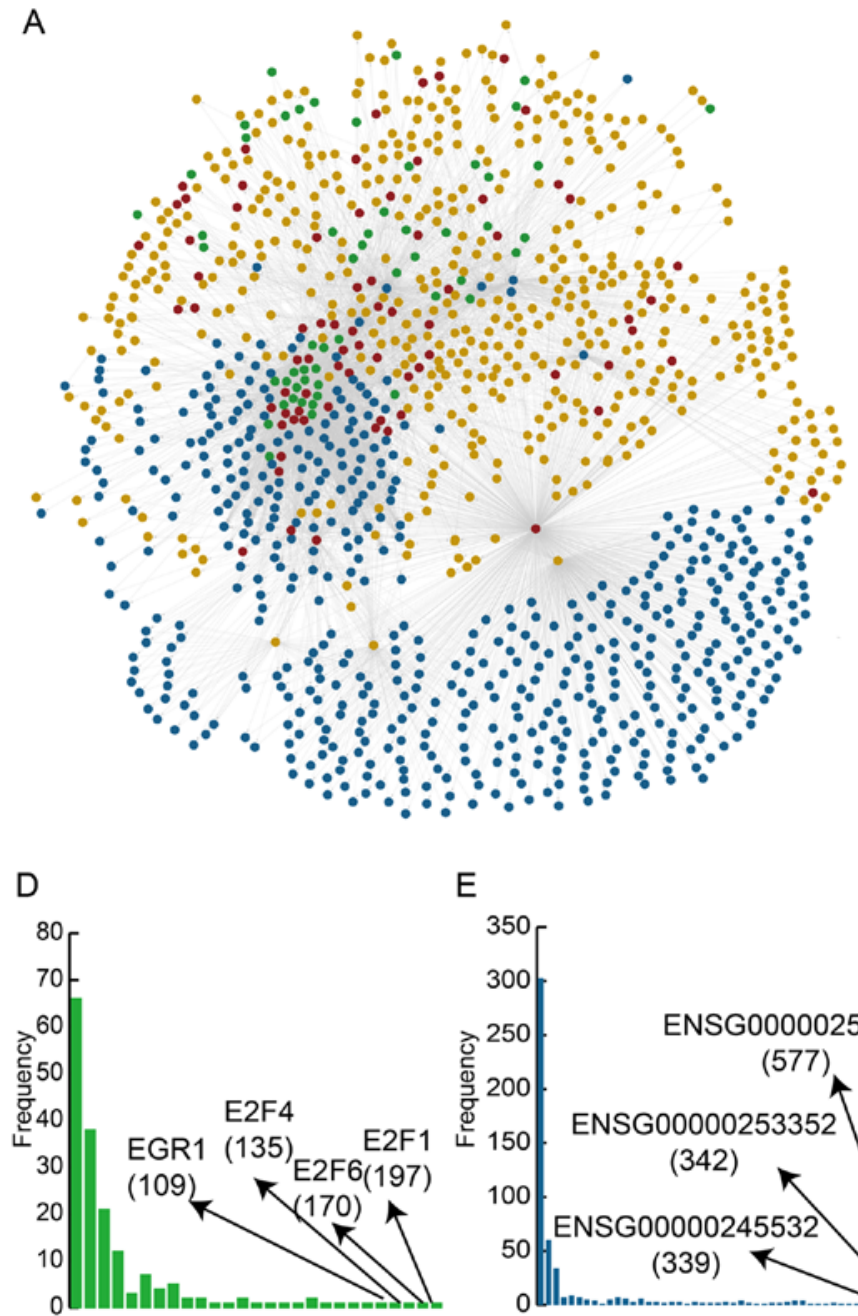

$\mathrm{E}$

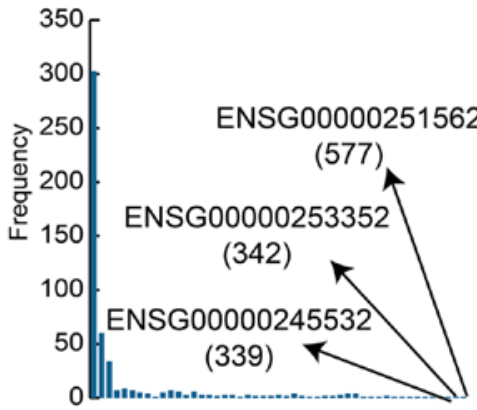

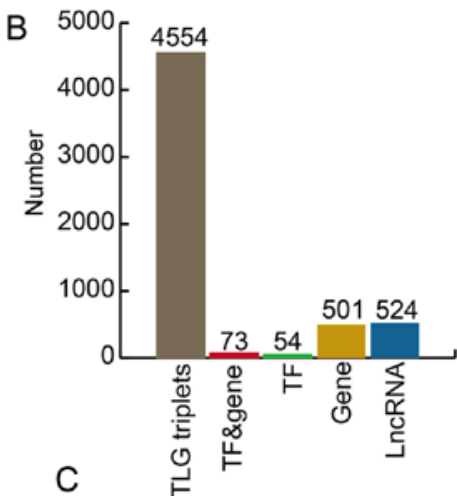
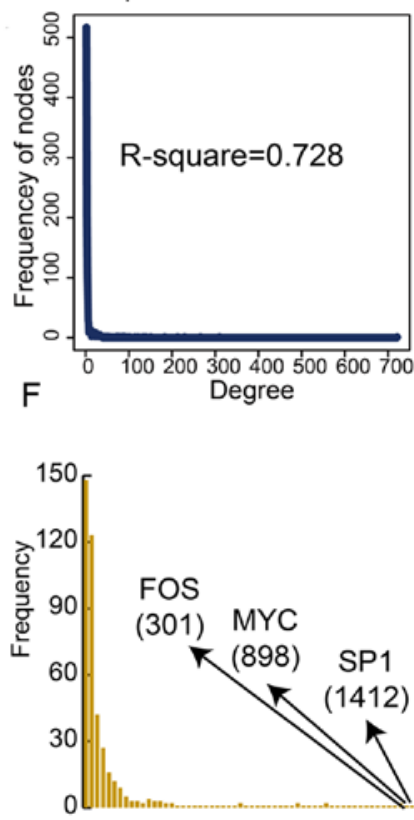

Figure 1. Construction and global characteristics of the global LncMRT network. (A) TFs and genes, TFs, genes, and lncRNAs are colored by red, green, orange and blue, respectively. (B) The number of molecules in the global LncMRT network. (C) The degree distribution of the global LncMRT network. The degree distribution of (D) TFs, (E) genes and (F) lncRNAs. TF, transcription factor; lncRNA, long non-coding RNA; LncMRT, IncRNA-mediated regulatory triplet; MYC, myc proto-oncogene protein; SP1, transcription factor Sp1.

The integrated core cluster could split all samples into diverse groups. A total of four groups were identified, according to the $\mathrm{CDF}$ and relative change in area under the $\mathrm{CDF}$ curve plot (Fig. 4C and D). Each sample group had a consensus expression pattern and could be distinguished clearly (Fig. 4E). Most samples could be classified accurately (Chi-square test, $\mathrm{P}=0.035$ ); this was especially true for the last group (C4), in which all matched control samples were verified (Fig. 4F). The first core cluster could also distinguish IS patient and matched control samples (Chi-square test, $\mathrm{P}=0.027$ ). Collectively, all the above results indicated that integrating the expression profiles of dysregulated LncMRTs may identify specific biomarkers that distinguish IS patient and matched control samples.

Dysregulated LncMRTs in IS patients are associated with critical biological functions and the PI3K/Akt signaling pathway. The GO enrichment analyses were performed based on all TFs and genes in dysregulated LncMRTs in IS. These genes were slightly enriched in some critical biological functions such as positive regulation of transcription, creating DNA templates, positive regulation of gene expression and positive or negative regulation of mesenchymal cell proliferation (Fig. 5A).
Mesenchymal cells were associated with recovery from IS and provided neurological protection (26). In addition, KEGG pathway enrichment analyses were also performed and several key pathways were enriched, such as the PI3K/Akt signaling pathway, the advanced glycation endproducts (AGE)-receptor for AGE signaling pathway, the estrogen signaling pathway and the tumor necrosis factor signaling pathway (Fig. 5B). The PI3K/Akt signaling pathway is an intracellular signaling pathway important in regulating the cell cycle. Quercetin can decrease cell apoptosis in IS rat brains and the mechanism may be related to the activation of the PI3K/Akt signaling pathway (27). The PI3K/Akt signaling pathway contributes to neuronal survival after IS (28). In the current study, 19 TFs and genes in the dysregulated LncMRTs were involved in this pathway (Fig. 5C). For example, PTEN is a key gene in the $\mathrm{PI} 3 \mathrm{~K} / \mathrm{Akt}$ signaling pathway and was also found to be a negative regulator of neuronal cell survival (29). PTEN formed a dysregulated LncMRT with HOX Transcript Antisense RNA (HOTAIR) and transcription factor AP-2- $\alpha$ (TFAP2A). In summary, these results suggest that LncMRTs associated with IS could play their roles by participating in the regulation of the PI3K/Akt signaling pathway. 


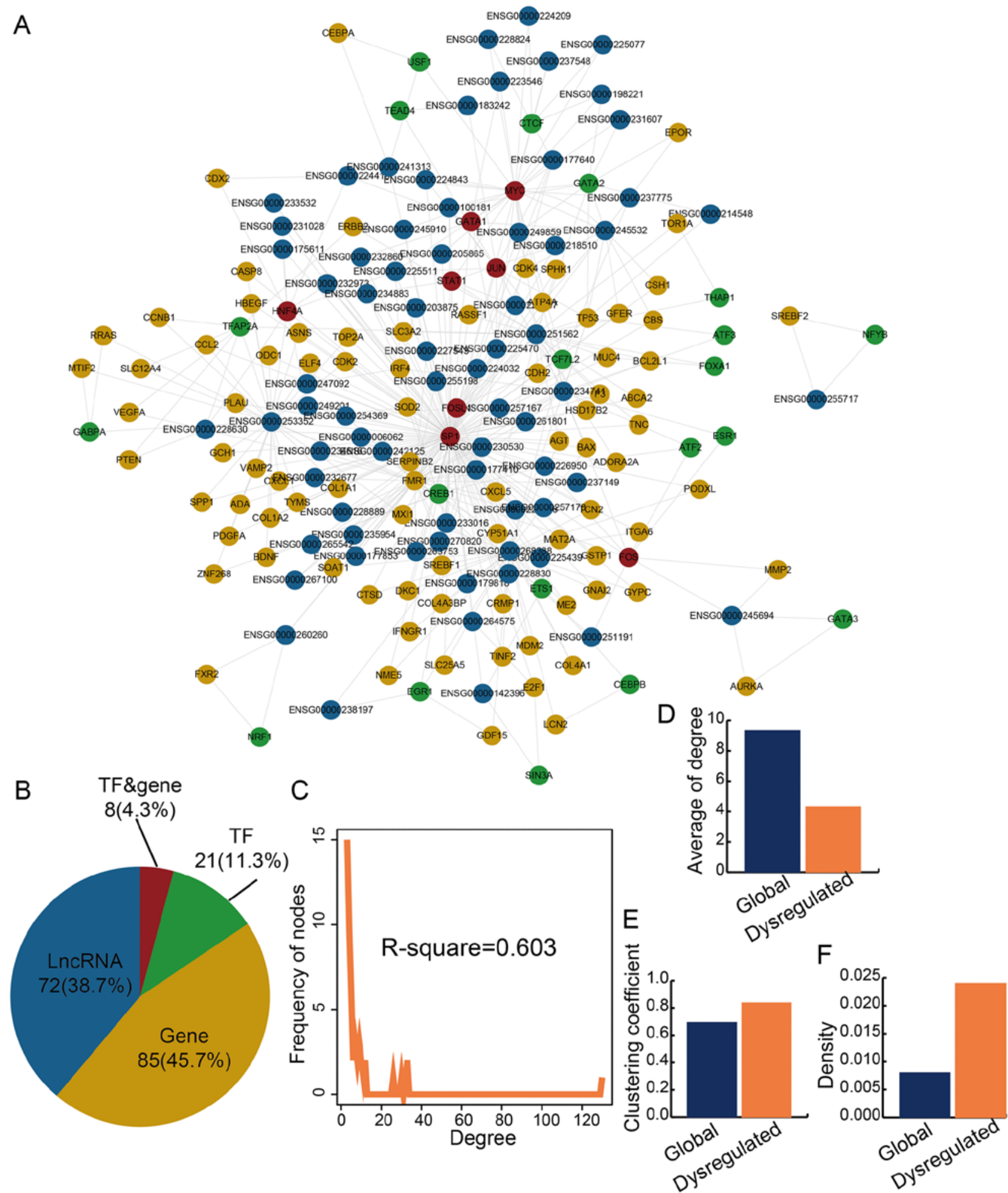

Figure 2. Dysregulated LncMRT network and topological features. (A) The dysregulated LncMRT network in ischemic stroke. (B) The percentage of TFs and genes, TFs, genes, and lncRNAs in the dysregulated LncMRT network. (C) The degree distribution of the global LncMRT network. Comparison of the (D) average degree, (E) clustering coefficient and (F) network density between the global LncMRT network and the dysregulated LncMRT network. TF, transcription factor; lncRNA, long non-coding RNA; LncMRT, lncRNA-mediated regulatory triplet.

\section{Discussion}

Detecting transcriptome changes is a major challenge of studying the mechanism of and treatment for IS. The expression levels of coding and non-coding transcripts were frequently regulated by each other. This cross-talk regulation among TFs, genes and lncRNAs could form regulated triplets, and may be functional motifs for IS. In the current study, an integrated and computational approach was developed using experimentally verified interactions and expression profiles of TFs, genes and IncRNAs to explore the LncMRTs in IS. 

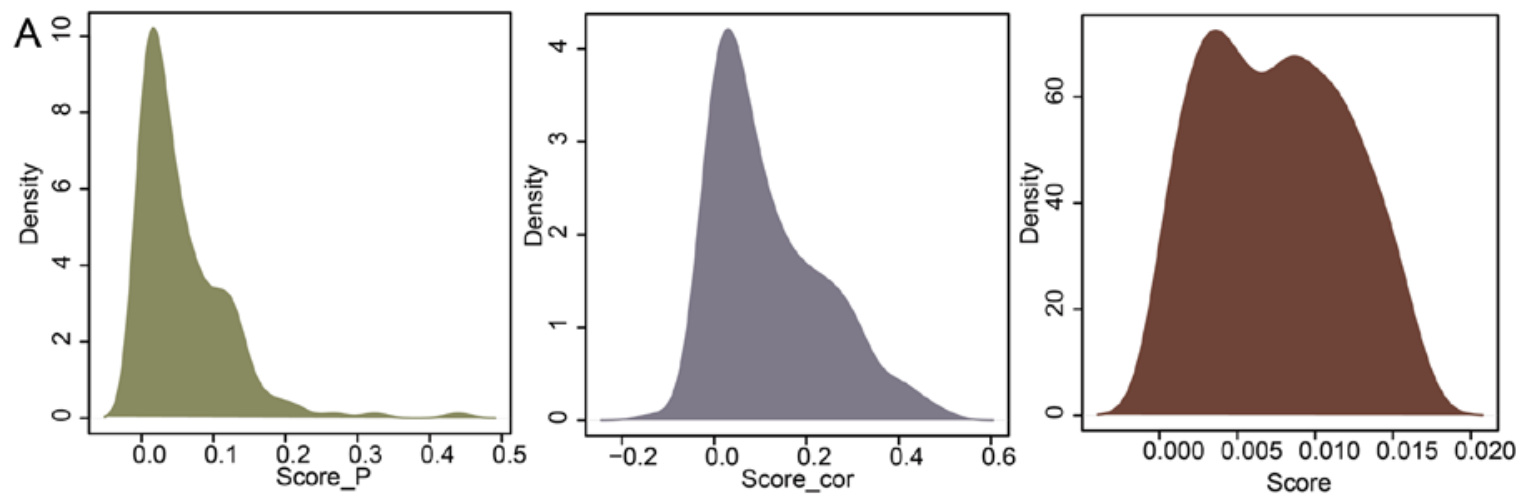

B

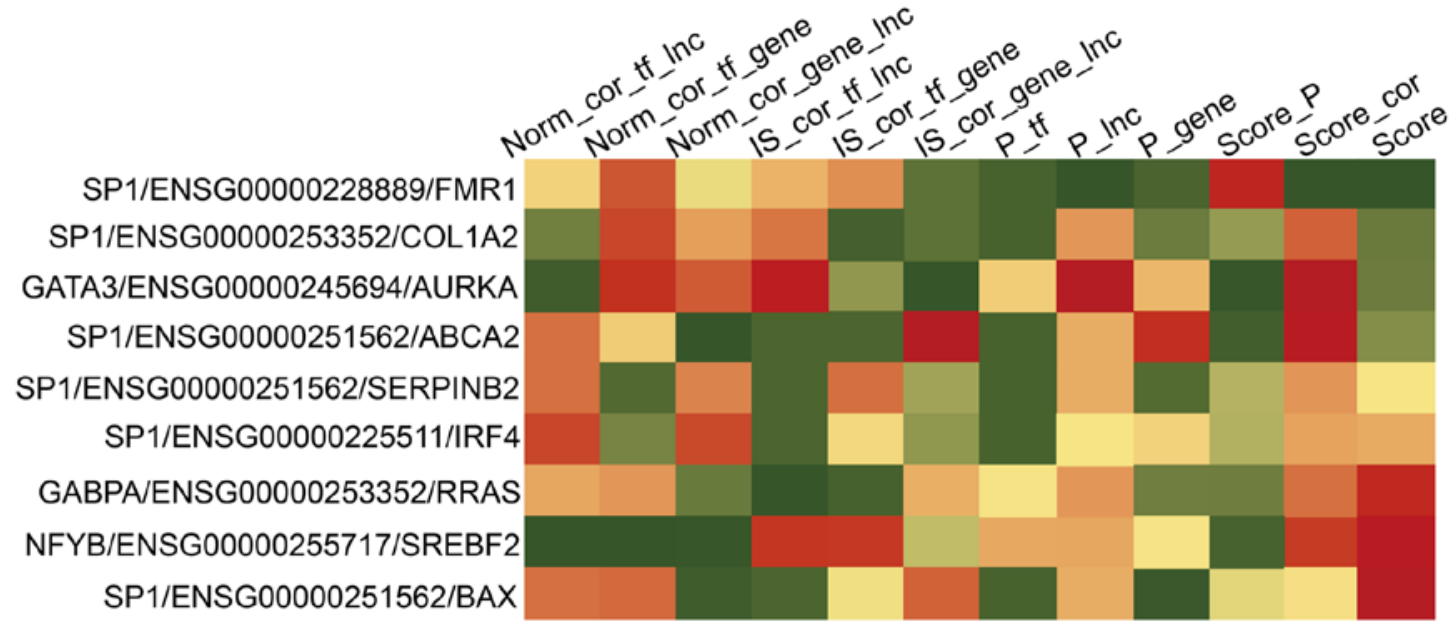

C
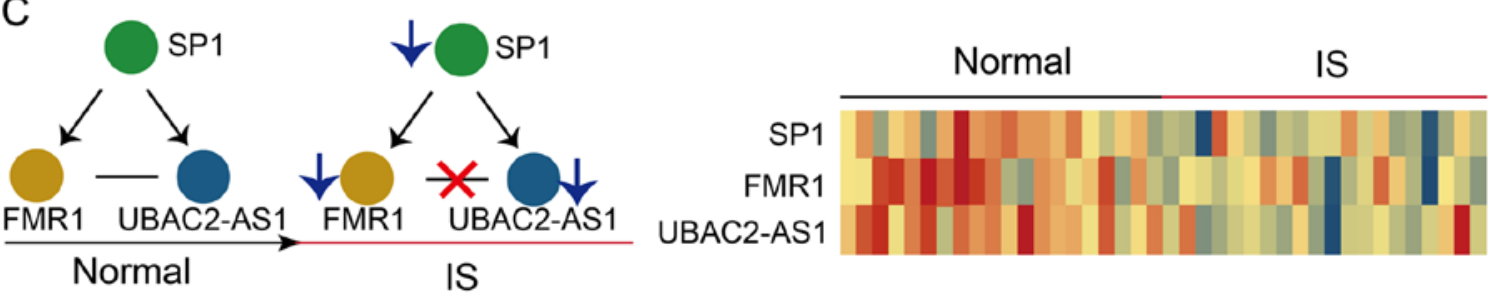

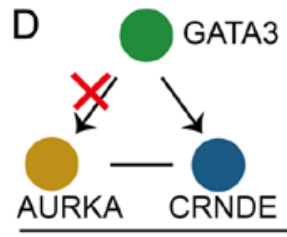

Normal
个○ GATA3
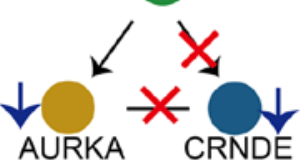

IS

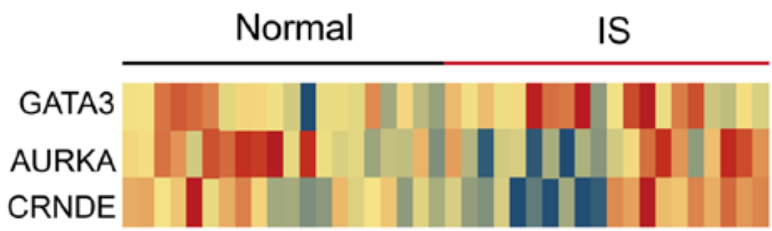

Figure 3. Construction of a functional score profile for the dysregulated LncMRTs in IS. (A) The density distribution curves of RSdif, RSPCC and integrated scores. (B) The activity score profile for the top dysregulated LncMRTs in IS. (C) The dysregulated patterns of LncMRTs (SP1/FMR1/UBAC2-AS1) in IS. The heatmap presented the expression of molecules. The crosses represent regulated interactions that were not established (no co-expression was observed). Red and blue arrows represent up- and downregulated transcription factors, lncRNAs and genes. (D) The dysregulated patterns of LncMRTs (SP1/FMR1/UBAC2-AS1) in IS (GATA3/AURKA/CRNDE). LncMRT, long non-coding RNA-mediated regulatory triplet; IS, ischemic stroke; RSdif, risk scores of differential expression P-values; RSPCC, risk scores of Pearson Correlation Coefficients; SP1, transcription factor Sp1; FMR1, synaptic functional regulator FMR1; GATA3, trans-acting T-cell-specific transcription factor GATA-3; AURKA, aurora kinase A; CRNDE, colorectal neoplasia differentially expressed; UBAC2-AS1UBAC2, antisense RNA 1.

In the current study, multiple dysregulated patterns emerged from the analysis of TF, gene and lncRNA expression, and variations in interactions in these LncMRTs. In some of the dysregulated LncMRTs, almost all molecules and interactions changed in IS patients compared with matched controls. In other dysregulated LncMRTs, only an individual molecule and interaction exhibited a clear change in IS patients. There were two major types of interaction change: i) The regulatory interactions were present in matched controls, but absent in IS patients; and ii) the regulatory interactions were absent in matched controls, but present in IS patients. Similar patterns and phenomena also appeared in other types of regulatory motifs, such as competing endogenous (ce)RNAs in cancer (30). Collectively, the results show that the regulatory interactions were absent or present in LncRMTs in IS patients compared with matched controls. 
A
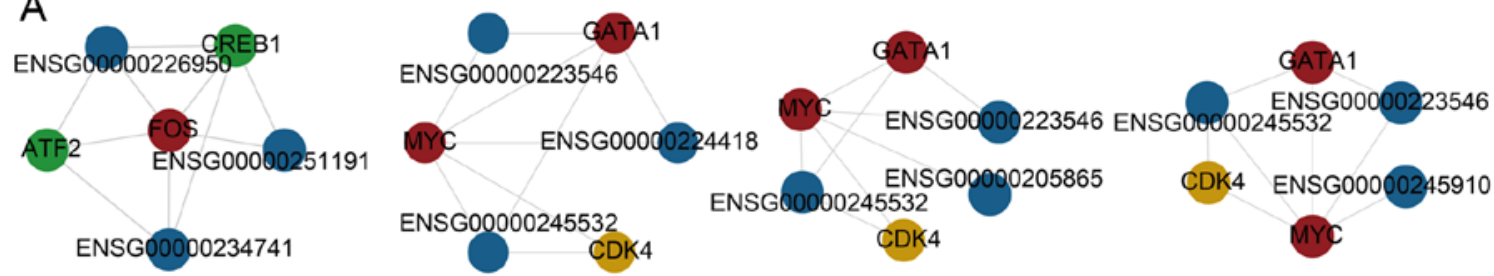

B

C
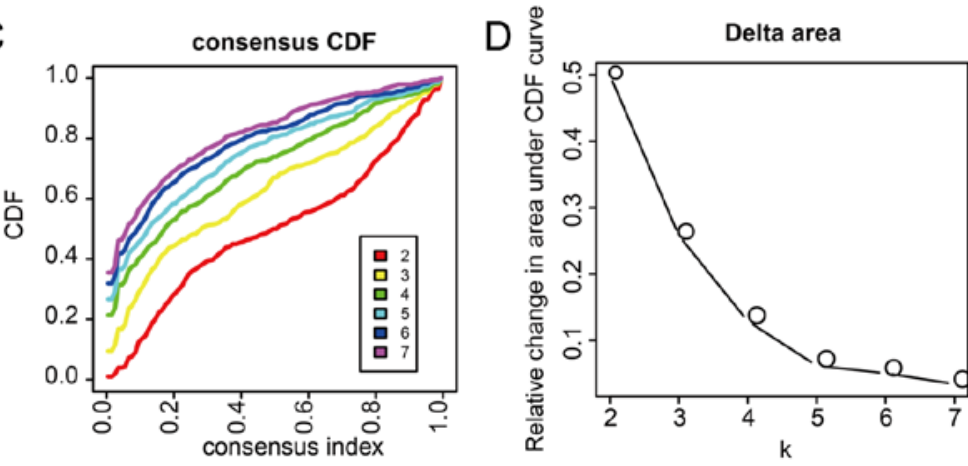

E
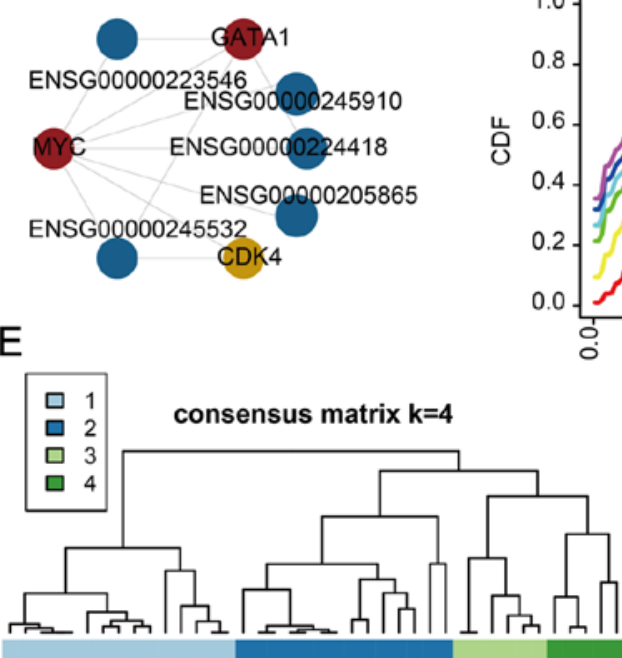

$\mathrm{F}$
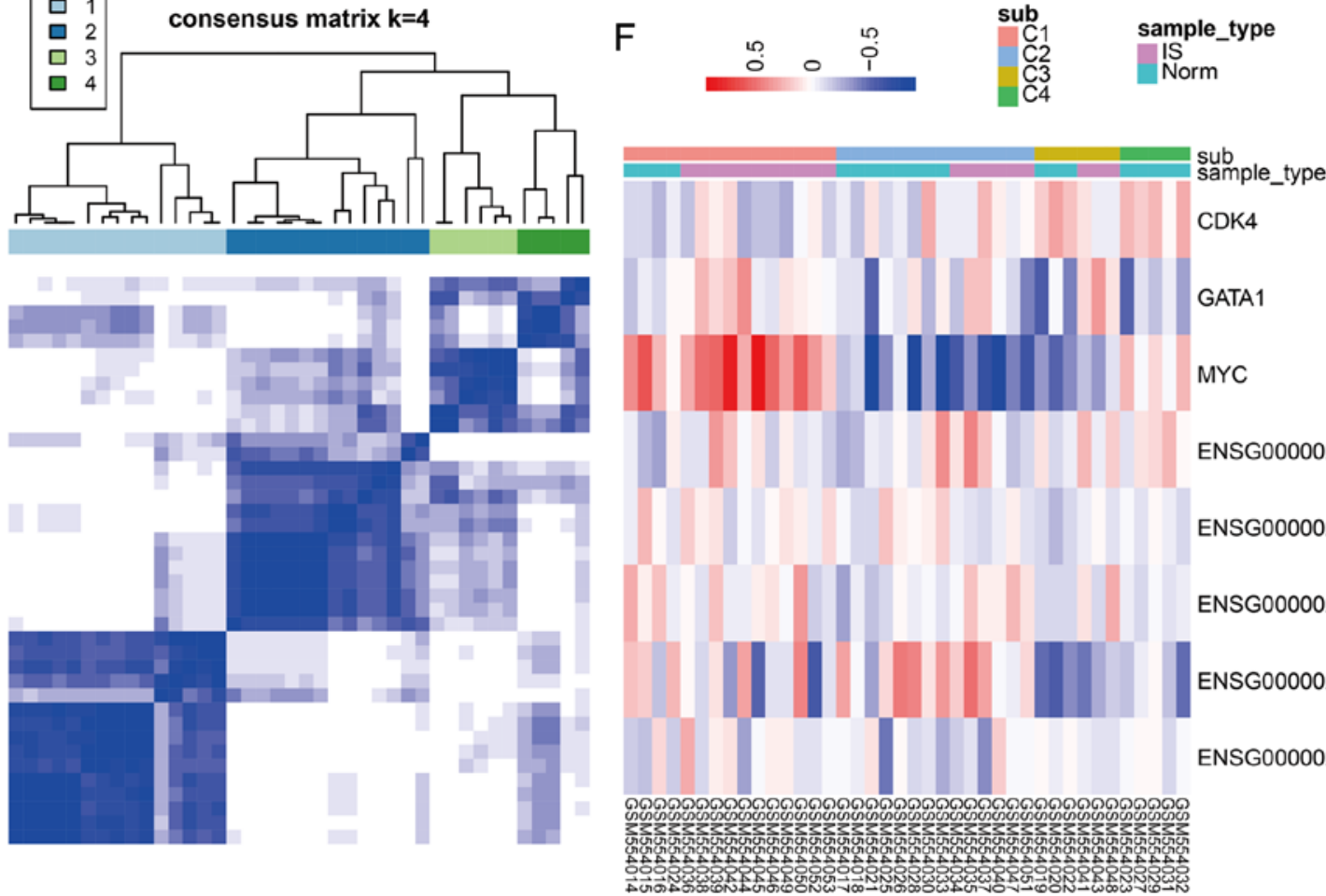

CDK4

GATA1

MYC

ENSG00000205865

ENSG00000223546

ENSG00000224418

ENSG00000245532

ENSG00000245910

Figure 4. Core clusters could distinguish ischemic stroke patient and matched control samples. (A) The core clusters identified from the dysregulated LncMRT network. (B) An integrated core cluster. (C) Cumulative distribution function plot of the consensus index. (D) Relative change in area under the CDF curve of different group numbers. (E) Consensus cluster heatmap of samples. (F) The gene expression heatmap. The sub-label refers to the group type classified by consensus cluster. Sample type refers to the disease status of the samples. LncMRT, long non-coding RNA-mediated regulatory triplet; GATA3, trans-acting T-cell-specific transcription factor GATA-3; CDK4, cyclin dependent kinase 4; MYC, myc proto-oncogene protein; CDF, cumulative distribution function.

The disturbance of these LncRMTs may contribute to the development of IS.

A previous study suggested that some other types of integrated and regulated motifs could be regarded as specific disease- and survival-associated biomarkers. For example, lncRNA-mediated ceRNAs could be viewed as prognostic biomarkers for breast cancer (31). In the current study, an integrated core cluster, including TFs GATA1 and MYC, the gene cyclin-dependent kinase 4 , and lncRNAs family with sequence similarity 99 member B, LINC00630, STK24-AS1, NEAT1 and small nucleolar RNA host gene 6 , was constructed. The integrated core cluster could split all the samples into four groups and may be a specific biomarker profile for IS. In addition, this study was undertaken to explore biomarkers in peripheral blood samples of IS patients and matched controls. The finding would be more meaningful if it could be tested in other types of samples, such as spinal cord fluid.

A key pathway named the PI3K/Akt signaling pathway was found by functional analyses of TFs and genes in the dysregulated LncMRTs of IS. The activation of PI3K correlates with increased cell survival and this effect is largely mediated through the activation of the serine/threonine kinase Akt (32). PTEN plays 
A

B

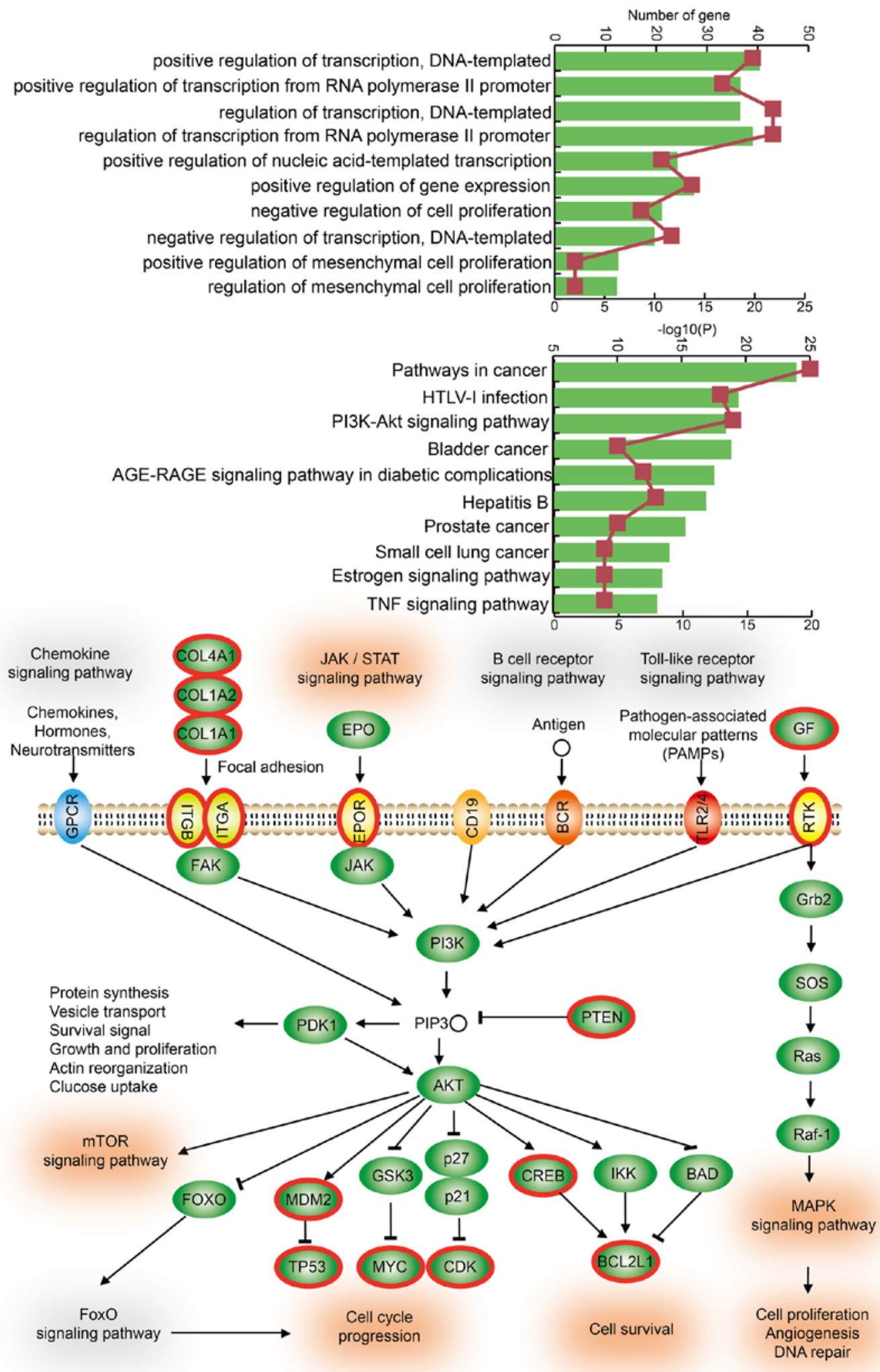

Figure 5. Functional analysis of transcription factors and genes in the dysregulated LncMRTs for IS. (A) GO terms and (B) KEGG pathways enriched for genes in dysregulated LncMRTs in IS, ranked by $-\log _{10}(\mathrm{P}$-value). (C) The PI3K/Akt signaling pathway and participating dysregulated LncMRTs. LncMRT, long non-coding RNA-mediated regulatory triplet; IS, ischemic stroke; GO, Gene Ontology; KEGG, Kyoto Encyclopedia of Genes and Genomes; PI3K, phosphatidylinositol 3 kinase; Akt, protein kinase B.

a key regulatory role in the cross-talk between the cell survival PI3K/Akt signaling pathway and the pro-death JNK signaling pathway, which raises a new possibility that agents targeting the phosphatase PTEN may expand the therapeutic options of agents used to protect neurons from IS (33). PTEN, TFAP2A and HOTAIR formed a dysregulated LncMRT in IS patients, which suggests that PTEN could play its role in the PI3K/Akt signaling pathway by forming LncMRT in IS patients. 
In conclusion, a dysregulated LncMRT network for IS was constructed and analyzed. The risk scores were used to evaluate the activity of each dysregulated LncMRT in IS. Multiple dysregulated patterns of LncMRTs in IS were also found. In addition, the core LncMRT cluster could distinguish IS patient and matched control samples, and could be viewed as a specific biomarker profile for IS. The functional analysis showed the association between dysregulated LncMRTs and the PI3K/Akt signaling pathway in IS patients. Collectively, the results of the present study provide novel insights into the mechanisms underlying the function of lncRNAs in IS.

\section{Acknowledgements}

Not applicable.

\section{Funding}

The current study was supported by the National Natural Science Foundation of China (grant nos. 81820108014, $81771361,81571166,81801190,81701190$ and 81701155), the applied technique research and development project of Harbin (grant no. 2016RAXYJ067) and the Postdoctoral project of Heilongjiang Province (grant no. LBH-Z17138).

\section{Availability of data and materials}

All data generated or analyzed during this study are included in this published article.

\section{Authors' contributions}

CYZ,ZHX, WLH and CLY conceived and designed the present study. CYZ, WJJ, LXY and KXT performed the experiments and analyzed the data. BCR, LS, LJ, SXS, WN and TK validated and improved the computational approach in the present study. CYZ and BCR wrote the manuscript. All authors read and approved the final manuscript.

\section{Ethics approval and consent to participate}

Not applicable.

\section{Patient consent for publication}

Not applicable.

\section{Competing interests}

The authors declare that they have no competing interests.

\section{References}

1. Deb P, Sharma S and Hassan KM: Pathophysiologic mechanisms of acute ischemic stroke: An overview with emphasis on therapeutic significance beyond thrombolysis. Pathophysiology 17 197-218, 2010.

2. Feigin VL, Lawes CM, Bennett DA, Barker-Collo SL and Parag V: Worldwide stroke incidence and early case fatality reported in 56 population-based studies: A systematic review. Lancet Neurol 8: 355-369, 2009.
3. Bejot Y, Caillier M, Ben Salem D, Couvreur G, Rouaud O, Osseby GV, Durier J, Marie C, Moreau T and Giroud M: Ischaemic stroke subtypes and associated risk factors: A French population based study. J Neurol Neurosurg Psychiatry 79: 1344-1348, 2008.

4. Quarta G, Stanzione R, Evangelista A, Zanda B, Sciarretta S, Di Angelantonio E, Marchitti S, Di Murro D, Volpe M and Rubattu S: A protective role of a cholesteryl ester transfer protein gene variant towards ischaemic stroke in Sardinians. J Intern Med 262: 555-561, 2007.

5. O'Connell GC, Petrone AB, Treadway MB, Tennant CS, Lucke-Wold N, Chantler PD and Barr TL: Machine-learning approach identifies a pattern of gene expression in peripheral blood that can accurately detect ischaemic stroke. NPJ Genom Med 1: 16038, 2016.

6. Vemuganti R: All's well that transcribes well: Non-coding RNAs and post-stroke brain damage. Neurochem Int 63: 438-449, 2013

7. St Laurent G, Wahlestedt C and Kapranov P: The landscape of long noncoding RNA classification. Trends Genet 31: 239-251, 2015.

8. He W, Wei D, Cai, Chen S, Li S and Chen W: Altered long non-coding RNA transcriptomic profiles in ischemic stroke. Hum Gene Ther 29: 719-732, 2018.

9. Zhu R, Liu X and He Z: Long non-coding RNA H19 and MALAT1 gene variants in patients with ischemic stroke in a northern Chinese Han population. Mol Brain 11: 58, 2018.

10. Hou XX and Cheng H: Long non-coding RNA RMST silencing protects against middle cerebral artery occlusion (MCAO)-induced ischemic stroke. Biochem Biophys Res Commun 495: 2602-2608, 2018.

11. Latchman DS: Transcription factors: An overview. Int J Biochem Cell Biol 29: 1305-1312, 1997.

12. Jiang H, Li T, Qu Y, Wang X, Li B, Song J, Sun X, Tang Y, Wan J, Yu Y, et al: Long non-coding RNA SNHG15 interacts with and stabilizes transcription factor Slug and promotes colon cancer progression. Cancer Lett 425: 78-87, 2018.

13. Sawaya AP, Pastar I, Stojadinovic O, Lazovic S, Davis SC, Gil J, Kirsner RS and Tomic-Canic M: Topical mevastatin promotes wound healing by inhibiting the transcription factor $\mathrm{c}-\mathrm{Myc}$ via the glucocorticoid receptor and the long non-coding RNA Gas5. J Biol Chem 293: 1439-1449, 2018.

14. Yu Y, Zhang M, Wang N, Li Q, Yang J, Yan S, He X, Ji G and Miao L: Epigenetic silencing of tumor suppressor gene CDKN1A by oncogenic long non-coding RNA SNHG1 in cholangiocarcinoma. Cell Death Dis 9: 746, 2018.

15. Sepe R, Pellecchia S, Serra P, D'Angelo D, Federico A, Raia M, Cortez Cardoso Penha R, Decaussin-Petrucci M, Del Vecchio L, Fusco A and Pallante P: The long non-coding RNA RP5-1024C24.1 and its associated-gene MPPED2 are down-regulated in human thyroid neoplasias and act as tumour suppressors. Cancers (Basel) 10: E146, 2018.

16. Zhou L, Xu DY, Sha WG, Shen L and Lu GY: Long non-coding RNA MALAT1 interacts with transcription factor Foxo1 to regulate SIRT1 transcription in high glucose-induced HK-2 cells injury. Biochem Biophys Res Commun 503: 849-855, 2018.

17. Ning S, Zhao Z, Ye J, Wang P, Zhi H, Li R, Wang T, Wang J, Wang L and Li X: SNP@lincTFBS: An integrated database of polymorphisms in human LincRNA transcription factor binding sites. PLoS One 9: e103851, 2014.

18. Wingender E, Dietze P, Karas H and Knuppel R: TRANSFAC: A database on transcription factors and their DNA binding sites. Nucleic Acids Res 24: 238-241, 1996.

19. Yi Y, Zhao Y, Li C, Zhang L, Huang H, Li Y, Liu L, Hou P, Cui T, Tan P, et al: RAID v2.0: An updated resource of RNA-associated interactions across organisms. Nucleic Acids Res 45: D115-D118, 2017.

20. Krug T, Gabriel JP, Taipa R, Fonseca BV, DominguesMontanari S, Fernandez-Cadenas I, Manso H, Gouveia LO, Sobral J, Albergaria I, et al: TTC7B emerges as a novel risk factor for ischemic stroke through the convergence of several genome-wide approaches. J Cereb Blood Flow Metab 32: 1061-1072, 2012.

21. Wilkerson MD and Hayes DN: ConsensusClusterPlus: A class discovery tool with confidence assessments and item tracking. Bioinformatics 26: 1572-1573, 2010.

22. Kuleshov MV, Jones MR, Rouillard AD, Fernandez NF, Duan Q, Wang Z, Koplev S, Jenkins SL, Jagodnik KM, Lachmann A, et al: Enrichr: A comprehensive gene set enrichment analysis web server 2016 update. Nucleic Acids Res 44: W90-W97, 2016. 
23. DeGregori J and Johnson DG: Distinct and overlapping roles for E2F family members in transcription, proliferation and apoptosis. Curr Mol Med 6: 739-748, 2006.

24. Chen S, Wang M, Yang H, Mao L, He Q, Jin H, Ye ZM, Luo XY, $\mathrm{Xia}$ YP and Hu B: LncRNA TUG1 sponges microRNA-9 to promote neurons apoptosis by up-regulated $\mathrm{Bcl} 2111$ under ischemia. Biochem Biophys Res Commun 485: 167-173, 2017.

25. Zhang X, Tang X, Liu K, Hamblin MH and Yin KJ: Long noncoding RNA Malat1 regulates cerebrovascular pathologies in ischemic stroke. J Neurosci 37: 1797-1806, 2017.

26. Gutiérrez-Fernández M, Rodríguez-Frutos B, Alvarez-Grech J, Vallejo-Cremades MT, Expósito-Alcaide M, Merino J, Roda JM and Díez-Tejedor E: Functional recovery after hematic administration of allogenic mesenchymal stem cells in acute ischemic stroke in rats. Neuroscience 175: 394-405, 2011.

27. Yao RQ, Qi DS, Yu HL, Liu J, Yang LH and Wu XX: Quercetin attenuates cell apoptosis in focal cerebral ischemia rat brain via activation of BDNF-TrkB-PI3K/Akt signaling pathway. Neurochem Res 37: 2777-2786, 2012.

28. Zhao H, Sapolsky RM and Steinberg GK: Phosphoinositide3-kinase/akt survival signal pathways are implicated in neuronal survival after stroke. Mol Neurobiol 34: 249-270, 2006.

29. Shi GD, OuYang YP, Shi JG, Liu Y, Yuan W and Jia LS: PTEN deletion prevents ischemic brain injury by activating the mTOR signaling pathway. Biochem Biophys Res Commun 404: 941-945, 2011.
30. Wang P, Ning S, Zhang Y, Li R, Ye J, Zhao Z, Zhi H, Wang T, Guo $Z$ and Li X: Identification of lncRNA-associated competing triplets reveals global patterns and prognostic markers for cancer. Nucleic Acids Res 43: 3478-3489, 2015.

31. Fan CN, Ma L and Liu N: Systematic analysis of lncRNA-miRNA-mRNA competing endogenous RNA network identifies four-lncRNA signature as a prognostic biomarker for breast cancer. J Transl Med 16: 264, 2018.

32. Kohn AD, Takeuchi F and Roth RA: Akt, a pleckstrin homology domain containing kinase, is activated primarily by phosphorylation. J Biol Chem 271: 21920-21926, 1996.

33. Zhang QG, Wu DN, Han D and Zhang GY: Critical role of PTEN in the coupling between PI3K/Akt and JNK1/2 signaling in ischemic brain injury. FEBS Lett 581: 495-505, 2007.

This work is licensed under a Creative Commons Attribution-NonCommercial-NoDerivatives 4.0 International (CC BY-NC-ND 4.0) License. 